\title{
Environmental turn in running the rural territories: new social technologies of administration
}

\author{
Konstantin Olkhovikov ${ }^{l}$, Svetlana Olkhovikova ${ }^{l}$, Lyudmila Zhuravleva ${ }^{2}$, Nisha Kant \\ $\mathrm{Ojha}^{3}$, Elena Zarubina ${ }^{2}$, and Aleksey Ruchkin ${ }^{2 *}$ \\ ${ }^{1}$ Ural Federal University named after the first President of Russia B.N. Yeltsin, Department of \\ Sociology and Technology, 620002, Mira street, 19, Yekaterinburg, Russia \\ ${ }^{2}$ Ural state agrarian University, Institute of Economics, Finance and Management, 620075, Karl \\ Liebknecht street, 42, Yekaterinburg, Russia \\ ${ }^{3}$ Sharda University, Department of Information Technology \& Business Analytics, 201310, Plot No. \\ 32-34, Knowledge Park III, Greater Noida, Uttar Pradesh, India
}

\begin{abstract}
Global warm-up makes the political agenda on highest levels. However, the science should make itself publicly heard, and not in the part of fundamental studies of nature only, but in the part of social science and social research of administrative tools as well. We offer three circles of quest that attract numerous scholars in the science of management and administration touching the context of complementing new ecological imperatives in running the rural territories: (1) the relevance of new environmental mentality to practical principles of existing rural administrations, (2) the communication challenges, opportunities, and risks in introducing new qualitative criteria into existing social technologies, which dominate modern rural administration patterns, (3) the importance of alternative in technological development of modern rural territories with a high agrarian overload, including the managing of alternative energy introduction, and trial of unprecedented technological schemes.
\end{abstract}

\section{Introduction}

Popular characteristics of modern, or even post-modern civilization as informational one, accentuates the informational resources and communications, which can superficially mislead to under-evaluation of material resources production, and last not least the agriculture with the food supplies. Nevertheless, what makes sense is the outreaching velocity in informational and mediating tools and resources that changes key indicators for human resources development. Here we argue that communication is not the opposite of agricultural development; moreover, the latter should not be the peripheral phenomenon of information age progresses. Agriculture knowledge management is on the rise [An organization of Ministry of Agriculture, Government of India, 2020] and gives good opportunities to human civilization, opening up and giving birth to the most pioneering,

${ }^{*}$ Corresponding author: alexeyruchkin87@gmail.com 
innovative, user friendly and self-supporting agricultural management institutes in the world, which demands a development of various and qualitatively unprecedented social technologies. Obviously, it comes with the environmental denominator of all actual challenges for human civilization, also bringing to the frontstage the human selfdetermination and prospective confidence in humanity survival. Environmental issues payback the values of traditional human practices, equally clearing the space for unprecedented solutions. While technologies discover the previously neglected features of objects and natural surrounding as a self-sufficient factor, the social technologies invent and re-discover human properties and overlooked abilities that could bring unprecedented social advantages. People bring their social situation to the environment, not vice versa; the unlearnt lessons of discovering and inhabiting new continents, institutionalizing the new world, stresses the actual demand for people-engaging solutions re-establishing old traditional ties with natural human environment, but this time through information and communication as the reigning mediators. Social technology is not the shadow of team building, no matter how important the latter is; social technology brings new frames of human responsible existence that brings close territorial and environmental awareness to communicative actions to optimize human experiences in the face of changing globality. The very term "effective rural communication for development" is clearly positioned as inclusive, not exclusive; "Participatory methods are tools to involve partners with each other, meaning that they are themselves communication tools", which does not mean alienation of information data, rather the opposite, when the belonging to the situation excludes the extortion of creative subjects [FAOUN, 2006]. Obviously, when the process of rethinking the agricultural extension gains the momentum, the frames for extra-dominant challenges are clearer drawn, and that is rooted in the "ever-changing social and natural environment" [Leeuwis, 1988]. People have more chances to talk to their true selves when staying outside the hustle and bustle of urban life that includes not only the external machinery, but also the internal mechanics of bureaucratic abusive power and marginalized marketing manipulations. However, getting back to the roots is not an easy excursion, as it presumes numerous interactions with global challenges being brought to the doorsteps of core peasant activities. As it is widely and confidently acknowledged that: "Agriculture is increasingly becoming more knowledge-intensive." [FAOUN, 2016]. Multiplying the emulating factors for farming is not a matter of marketing survival, rather the introducing of new options and opportunities for new generations of people, provided we adequately comprehend and cope with the challenges initiated by the civilizational complexity of the actual agriculture social technologies. Moreover, ICT is the key tool to positive development of successful decision-making [Kamthania, 2016], changing the farmer's perspective of cropping, but also reformatting the bureaucratic prescriptions running the eternal normative pressure on rural developments. Big data is important when it brings us to the improvement and optimization of human-to-human daily transactions; clouds and online procedures all lead to offline human existence that is still preceding the probable human essence, as the twentieth-century French philosopher jean-Paul Sartre argued.

Romantic reading of environmentalism is preoccupied with taking our imagination to idealized in their tranquility rural areas; so, what is the use of bringing the most advanced technologies to overloaded modern rural territories, if we aim at alleviating and substantially improving their life chances and leap over from surviving to attractive prosperity in its corresponding to progressively developing nature of human social self? We find the optimal formula to answer the challenge in two steps. Firstly, environment is the process, not the reified objectivity, while human activity, being essentially social is also much more fruitful when propagating communicative tactics; thus, communication brings us to the winning environmental strategy due to evading of direct strategical pressure of objectified efficiency challenges, and relying mostly on human inclination to socialize in a 
peaceful and secure way, when life is the true goal in itself, not an instrument to external achievements. Secondly, since the genial idea of Ferdinand Tönnies that consisted in consequential juxtaposing of 'Gemeinschaft und Gesellschaft' had been dubbed in twentieth-century American sociology 'a cultural theorem', we find it excusable to press a little bit further in interpreting the interpretation of community / society distinction not a static essence, but as existing process initiation that lies in the human ability to act in awareness of one's belongings in their historical transformation in local and global contexts simultaneously. More non-violent communicating brings us closer to environmental harmony which is not a utopia.

\section{Methodology}

Making a social comparative study, we always come to facing numerous offline variations in social practices that perform the essentiality of rural way of life, mostly the folkways linked to intermediate human contacts and communications. People are being unequivocally readdressed to themselves as "the emerging picture of climate change, while complex, is increasingly comprehensible" [Keller, 2001]. Actual social situation, as well as individuals' abilities to evaluate the one, a ubiquitously influenced by Internet data and online forms of representation, which includes the local facts and events. As we know, the best examples of social surveys were accomplished with the simplest tools, starting with a relaxed human conversation, which is almost unattainable luxury. Nevertheless, the Internet online survey is here to compensate the lack of sociable time in the most expectable form of an online chat. Thus, we move to reconstructing the social situation with a mixture of online and offline social contacting, and a proportion of qualitative and quantitative data. Now it is democratic and available tool of Internet survey to open the social situation with environmental awareness, with cleaner sources of energy, and with perspectives of socially responsible entrepreneurship in rural territories that are leaving the offline status for good.

We can also dub the tool as a multi-dimensional 'delegated interview' (the formula well-known to the famous researcher Valery Tishkov, in his study of the last Chechen war). We argue new constructive principles for Indian-Russian project in social science rural research that start from the basics of sociological imagination, go through comparative qualitative analysis correlating with big data quantitative models, to case projecting that reinterprets the borders between social psychological typologies, rural administrative management, and intra-disciplinary rural territorial development projects.

Surely, doing a comparative study can only put us deeper into the stock-piled data; however, we see the priority in qualitative judgements, which means accentuating the language of actual studies, bringing it to the state of transparency from the quotidian praxis points of view, i.e., the primary human experiences and judgements, reflected by numerous social researchers in the field and in different parts of the globe. In fact, strategic communication is an institutionally acknowledged social tool to be spread in all countries and for the whole humanity [Santucci, 2005]. We also share the inspirations of various international research groups initiating fresh and stunningly long-missed comparisons of rural experiences [Ponniah et al., 2008].

Moreover, there are no questions with adequate solutions without a comparative international study of social life specially the rural one, we are but supporting the mainstream trend of global cognitive developments: "The rise of digital agriculture could be the most transformative and disruptive of all the industries, because digital agriculture not only will change how farmers farm their farms, but also will transform fundamentally every part of the agri-food value chain.”[ Trendov, N. M., Varas, S. \& Zeng, M., 2019]

Looking for exemplary cases does not mean stricter selection for raw qualitative phenomena, with but one exclusion, as we moving towards things as they are in their 
probable amelioration, not towards ideal semantic schemes that do not invade the sovereignty of naïve attitudes running existing social practices and social institutions. Accepting key foundations of Karl Popper's critical rationalism, we will be the last ones to support the stereotypical endeavor of extreme empiricist who still believe in 'facts speaking for themselves'. Still, absolutely relativist and comparative qualitative investigation can and should lead to absolutely critical principles for constructing new social technologies frame focused on communicative improvements, when combined with open intradisciplinary case solutions (when research diffuses to a project and backwards).

\section{Rural territory as a cradle of human race}

As Eisenstadt strongly argues, "the emergence of one such worldwide civilization, with basically only local variants" [Eisentadt, 1989] becomes irrelevant nowadays. In fact, the stronger link between communication and human lifeworld uncovers the primordial cultural status of landscape, to say nothing of culture as being the derivation of agriculture. The socalled network society produces and reintroduces its own internal counter-powers which are not purely symbolic [Castells, 2007], being mostly the pragmatic ones. Anyhow, the human pragmatics did not start from the office as it had preceded the bureaucratic developments in local communities. The arable land is the ground for making food and feeding the human traditions, which are always local in their global spread.

Social regulation is a complex, multi-level, and heterogeneous aggregate that is inclined to represent itself systematically in the minds of the controlled individuals. That means the inevitability of irrational breakdowns in norms belonging to social regulation. Nevertheless, rationality, instrumental control, and correctness as the highest criterion are kept up as the frame options for every existing organization that answers the actual challenges of modernity. As we know, the influential theory of communicative action propagates a realistic vision of traditional legacy, which "offers us a frame of reference which may help us to reflect on both the points of connection and the discontinuities between the past and our current predicament" [Habermas, 2005].

Human race comes from the rural communities, never forgetting the unique primary experiences of survival that form cultural styles of pragmatics, including communicative practices. Taking into account the fundamental fact of internet and mobile nets integration, we should but confirm the dissolution of such notions and phenomena as 'efficient centralized controlling' and 'periphery'; the clock-like mechanical prototypes of social administration are irrelevant and only partially supported by obsolete managerial practices still included in social technologies.

"The mission of e-Agriculture is to facilitate the discussion on the adoption and use of ICTs and digital innovations in agriculture, forestry, fisheries, natural resource management and rural development." [FAOUN] The global choice is loud and clear, therefore we need to clarify some basics in the light of modern social theory opportunities, no matter how differentiated and segmented the actual social knowledge is. Anyway, starting with the elementary, every author for the theme, which is also ours, should begin with some generalized premises that should reflect relevant vision of existing human situation in the global age, which is also informational, and with huge environmental challenges. Still, our attitude starts with a vision of human communication that essentially incorporates the rural roots of all viable social communities.

French philosopher Rousseau was not far from truth indicating the irreversibility of civilizational morality in the context of individuals social sentiments. However, the adoption of long-lost human practices should nurture not the ethnocentric romanticism only. Certainly, the living cultures differ in their representation of cultural universalia, 
nonetheless reintroducing the variative approach to human survival, where the invariant component that reflects human cognitive mission, becomes social one as well.

Actual public discussions on food safety are bringing once again the past and present to the futuristic solutions of essential problems of human survival, correlating wisdom and prosperity with affluence in ne efficiency terms [FSN Forum, 2016]

Social institutions are multifarious, including so-called informal ones. Meanwhile, he general build and overall frame for social norms of every level and scope is defined by social institution of morality that starts with manners and folkways, expresses itself in mores, coming up to official norms, specific codes, and in the end of the day touches the sphere of values, varying in attitudes and higher motives.

We are not looking for new stunning definitions of community, we neither look for exclusive characteristics of mostly successful community in the third millennium; we humbly but insistently introduce the pragmatic idea of culture as the primary, genuine, and absolutely inclusive polyvariant spontaneous 'social technology' of human survival, which relies on symbolic utterances in the process of communication as the universal and supercivilizational social institution. Cultural universalia are good for public policy declarations, but in fact, they live in endless local variations and through unique personal human experiences. Surely, economics is not an exclusion, and could not be hidden after management schemes that do not speak for themselves in the existing civilizational drift, caused by environmental challenges to humanity.

\section{Social technologies as alternative consequence of technological overload}

We start our life through learning, and we must relay our accumulations in the ameliorated form to the generations coming ahead. Symbols are not universal by their own, they rely on human lives. "Although it is legitimate to treat social relations - even relations of domination - as symbolic interactions, that is, as relations of communication implying cognition and recognition, one must not forget that the relations of communication par excellence - linguistic exchanges - are also relations of symbolic power in which the power relations between speakers or their respective groups are actualized. In short, one must move beyond the usual opposition between economism and culturalism, in order to develop an economy of symbolic exchanges" [Bourdieu, 1991]. Any social interaction is a type of process that presupposes at least two subjects, and in case of their administrative statuses, two decision-makers. However, decision-making is impossible without communication, but communication is just a tool in such cases. Thus, administrative interaction starts with communication that evaporates in the resulting perpetration of administrative functions. Moreover, planning, organizing, motivation, and controlling should be working only if their content is free from points of discussion. On the other hand, the efficiency evaluation orients the administrative functioning to back pay relations with objects of decision. Administrative interaction is either horizontal, closed and informal, or vertical, public, and formal. The interaction is a power secret, or an underdog of administrative decisionmaking. To make the administration more effective is to change the standard underevaluation of communication by some form of social technology that acknowledges the realities of the information civilization. No doubts, the communication could not replace the administration essentially, but the latter should change the essence of the former in context of new progressive developments in human civilization. Theory of communication comes closest to vindication in cases of rural administration, when powers exist only in interacting with other subjects and resources-holders to solve people's problems concerning the territorial infrastructure and social functioning. Human dimension of relatively autonomous life-worlds in our global world does not perpetrates the perspective fusion of 
online and offline resources automatically. Human resources crave for inhuman support of informational contacting and authoritarian manipulations to predict the grass-roots decision-making.

Here we come to the point where the daily human routine converts into innumerous actual communication practices and symbolic interactions creating and transforming new social institutions. No doubts, we need a broader understanding of the institutions that will include human lifeworld belonging and meaningful forms of human communities with their proper realistic landscapes. "Poverty does not belong in civilized human society. Its proper place is in a museum. That is where it will be." [Yunus Centre] Moreover, even microcrediting is not an evil, as it could be re-interpreted in intermediate social situations of communal human partnerships [Olkhovikov, Maruvada, 2018].

Anyway, "Management procedures indulge different methods and technologies unpredictable in relationships with organizational culture. Administrative and economic approaches usually do not catch the actual situation. Differentiation of organizational living worlds moves the pragmatic evaluations closer to social engineering." [Olkhovikov, Olkhovikova, 2016]. Social technology is not an objectified tool set, it grows through human capabilities realized through teamwork. Communities have the best resources for survival, especially the rural communities.

People do not rule, people deserve to be ruled for the better, joining the overall communal body of human race in various professional ways. Vocation is the last human social institution to die out (no more speculations). Anarchism is not far from the revelations of advanced human resources management, as it totally compensates the ambiguity of the term 'human resources' by introducing cognitive mistrust of formalizing social institutions and re0introducing the slogan "back to communities!".

Since old Greek myths are not about to loose their popularity, we find it acceptable to remind that we still share the legacy in opposing of total and differing 'dialectics', in its proper application to methods of discussion and to methods of cognition. After all, the potential of 'total dialectics' had been demonstrated in several spectacular and tragic ideologies during the last twenty-five centuries, so, what? The postmodernist replies heavily overlooked the irreplaceable analytical attitudes in their due social contexts, as they are irrelevant with totalitarianism, moreover, represent the unique antidote for the latter. Thus, turning our minds to future progressive developments, we cannot ignore the 'differentiating dialectics', but with one specific addition, - heterogeneity is not static, and not static ideal at all. As the relativity theory in physics did not introduced the relativity as an ideal, rather as a cognitive tool of high practical significance, the same is hopefully correct for the principle of heterogeneity is social sciences and social technologies.

\section{Conclusion}

The impact of communicative social technologies is not just an impulse, but a kind of multifarious developments partly invigorating the traditional resources of communal lifeworld that in fact never disappear but transform into some integrated practices of the digital era. Differentiating from authentic and mostly archaic traditions, these practices grow as a matter for advanced social technologies relying on environmental awareness. Village people have the shortest way for surviving the challenges of the third millennium. The millennials are undergoing the self-imposed social experiment in surviving through digital means while supporting the offline needs and wishes, which illustrates another aspect communicative action social theory, when "Symbolic meanings constitute or establish identity in a way similar to rules that establish unity in the multiplicity of their exemplary embodiments, of their different realizations or fulfillments." [Habermas, 1987]. When style, not hardware assembles, and shades, not overall tones define the outcome of 
the situation, the millennials get their chance for heading the actual human civilization. Decentralization is not a key player in modern public administration, however, the decentralization based on advanced digital technologies makes the people, namely, the citizens living on their land, the key players in the global world: "Vast new opportunities now exist to improve the quality and access of public service provision in developed and developing countries alike." [Schiavo-Campo, Sundaram, 2001]. Anyway, the humandimensioned development of digitalization in rural areas and farming production are acknowledged as a perspective for the human freedom globally [Freedom House, 2013].

As we declare our belonging to the future, we feel obliged to specify our initial projective steps in the direction. And yes, the paper is also an application to promote our Indian-Russian research project that starts with analyzing in the comparative trend the stories of modern Russian and Indian farmers, the rural entrepreneurs, belonging to the territories that surround big urban conglomerates; and the study that begins with expectable sociological data is aimed at producing pragmatic projects in the spheres of clean environment and modern communication brought together. The territories are New Delhi, India and Yekaterinburg, Russia. Above all, it is important those are not our final stop, but places of our initial location and true belonging.

\section{References}

1. Agriculture Knowledge Management, An organisation of Ministry of Agriculture, Government of India. URL: www.manage.gov.in

2. Framework on effective rural communication for development, Food and agriculture Organization of the United Nations (Rome, 2006)

3. C. Leeuwis, Communication for Rural Innovation. Rethinking Agricultural Extension Third Edition (Blackwell Science, 1988)

4. E-agriculture strategy guide: Piloted in Asia-Pacific countries, Published by the Food and Agriculture Organization of the United Nations and International Telecommunication Union Bangkok (2016)

5. D. Kamthania, ICT in Agriculture, (Indian Institute of Technology, Delhi, uploaded by Deepali Kamthania, 2016)

6. C.F. Keller, Global warming (Los Alamos National Laboratory branch of the University of California's Institute of Geophysics and Planetary Physics, 2001)

7. F.M. Santucci, Strategic Communication for Rural Development (World Bank Group, 2005)

8. A. Ponniah, R. Puskur, S. Workneh, D. Hoekstra, Concepts and practices in agricultural extension in developing countries: A source book, (International Livestock Research Institute, 2008)

9. N.M. Trendov, S. Varas, M. Zeng, Digital technologies in agriculture and rural areas - Status report, Licence: cc by-nc-sa 3.0 igo (Rome, 2019)

10. S.N. Eisentadt, Cultural Tradition, Historical Experience, and Social Change: The Limits of Convergence, The tanner lectures on human values, Delivered at The University of California, Berkeley, May 1-3, (1989)

11. M. Castells, International Journal of Communication, 1, 238-266 (2007)

12. J. Habermas, Democracy and the Public Sphere (Luke Goode, 2005)

13. Global Forum on Food Security and Nutrition, FSN Forum, Summary of the online discussion, 09.11.2016 - 30.11.2016, 134 (2016)

14. Food and Agricultural Organization of the United Nations. URL: www.fao.org/eagriculture/

15. P. Bourdieu, Language and Symbolic Power (Polity Press, 1991)

16. Yunus Centre site. URL: https://muhammadyunus.org/quotes 
17. K. Olkhovikov, V. Maruvada, Micro-financing for entrepreneurs in developing countries: Bangladeshi farmers case, ICERI 2018 Proceedings, 6909-6912 (2018)

18. K. Olkhovikov, S. Olkhovikova, Organizational myths: unconscious basics of organizational culture, The 10th International Days of Statistics and Economics. Conference Proceedings, September 8-10, 2016; Prague. Prague, Czech Republic, 1392-1399 (2016)

19. J. Habermas, The theory of communicative action, V. 2, Lifeworld and system: a critique of functionalist reason (Beacon Press, Boston, 1987).

20. S. Schiavo-Campo, P. Sundaram, To serve and to preserve: improving public administration in a competitive world (Asian Development Bank, 2001)

21. Freedom of the Net 2013. A Global Assessment of Internet and Digital Media. Freedom House (2013) 